\title{
Successful Treatment of stage IIlb intrahepatic Cholangiocarcinoma After Neoadjuvant Therapy With PD-1blockade Camrelizumab: A Case Report and Review of Literature
}

\section{Shuguang Zhu ( $\nabla$ za200909@163.com )}

Third Affiliated Hospital of Sun Yat-Sen University https://orcid.org/0000-0002-6939-9326

Haibo Li

Third Affiliated Hospital of Sun Yat-Sen University

Jianwen Zhang

Third Affiliated Hospital of Sun Yat-Sen University

Chunhui Qiu

Third Affiliated Hospital of Sun Yat-Sen University

Tianxing Dai

Third Affiliated Hospital of Sun Yat-Sen University

Hua Li

Third Affiliated Hospital of Sun Yat-Sen University

Guoying Wang

Guangzhou Medical College First Affiliated Hospital

\section{Research Article}

Keywords: Intrahepatic Cholangiocarcinoma, Lymph node Metastasis, Neoadjuvant Therapy, Immunotherapy, Chemotherapy, Surgical Resection

Posted Date: June 25th, 2021

DOI: https://doi.org/10.21203/rs.3.rs-348442/v1

License: (c) (i) This work is licensed under a Creative Commons Attribution 4.0 International License. Read Full License 


\section{Abstract}

Prognosis of ICC with lymph node metastasis is poor. And the feasibility of operation is uncertain, which is a contraindication in NCCN guidelines. In addition, in the neoadjuvant therapy of ICC, the role of immunotherapy is not clear. We describe a case of ICC with lymph node metastasis was successfully treated with neoadjuvant therapy.

\section{Background}

Intrahepatic cholangiocarcinoma (ICC) is an adenocarcinoma originated from the secondary bile duct and its branch epithelium. ICC accounts for about $10 \%-15 \%$ of primary liver cancer(1). ICC is the primary malignant tumor of liver after incidence rate of $\mathrm{HCC}(2)$. The incidence rate is increasing in recent years.

The exact cause of intrahepatic cholangiocarcinoma is still unclear. The well-known risk factors of ICC include congenital choledochal cyst, chronic cholangitis, chronic inflammatory bowel disease, primary sclerosing cholangitis, parasitic infection, chemical carcinogens (such as thorium dioxide and nitrosamine), genetic factors, biliary cirrhosis, cholelithiasis, alcoholic liver disease and non-specific cirrhosis. Studies have found that hepatitis virus is also closely related to ICC(3).

The general morphology of ICC can be divided into three types: mass type, peritubular infiltration type and intratubular growth type. The most common type is mass type, accounting for $60 \% \sim 80 \%$ of ICC; periductal infiltration type accounts for 15\% 35\%, which can diffuse infiltration along the biliary system and portal vein system, resulting in bile duct stenosis and peripheral bile duct dilatation; intraductal growth type accounts for $8 \%$ 29\%, which mostly shows papillary, polypoid or granular growth, spreading along the superficial bile duct(4). Here, we report the case of a patient with advanced ICC, which was successfully treated with neoadjuvant therapy combined with surgical resection.

\section{Case Presentation}

A 60-year-old man was referred because of "Right upper abdominal pain for 1 week". He lost weight about $2.5 \mathrm{KG}$ in the last 2 months. Abdominal physical examination was normal except for liver palmar and spider nevus because of hepatitis $B$ about 30 years. Mass in right lobe of liver were found by enhanced CT in other hospitals, which may be considered as ICC, and the right branch of portal vein is suspected to be involved (Fig. 1); and Hilar and retroperitoneal lymph node metastasis was found. No bone metastasis was found on ECT. Laboratory tests showed abnormality, CA19-9 is $1844 \mathrm{U} / \mathrm{mL}$ and HBV DNA is $6.0 \mathrm{e} 2 \mathrm{IU} / \mathrm{ml}$; other laboratory tests were normal, Including: AFP, CEA, CA125, ca15-3. It was initially diagnosed ICC (Stage IIIb) with hilar and retroperitoneal lymph node metastasis. Chemotherapy is recommended according to NCCN guidelines. So we did liver biopsy and pathological results showed poorly differentiated cholangiocarcinoma (Fig. 2).

According to AJCC disease stage / classification, intrahepatic cholangiocarcinoma with hilar lymph node

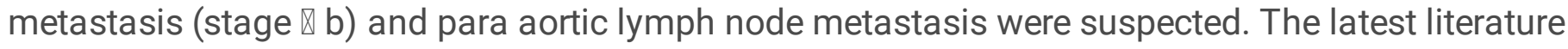


reports showed that two patients with stage $\triangle B$ ICC received $13 \mathrm{~m}$ and $16 \mathrm{~m}$ CR after chemotherapy with PD-1 monoclonal antibody. After multi-disciplinary consultation, we believe that patients should be given neoadjuvant therapy and surgical resection and lymph node dissection plus postoperative adjuvant therapy. After three times of PD-1 immunotherapy and two times of GP regimen chemotherapy, the tumor size of the patients was reduced, reaching the PR of oncology (Fig.3).

Furthermore, preoperative evaluation found that the patient has reached the condition of surgical resection(The Child Pugh grade of this patient is A, ICG R15 is $4.7 \%$, left liver volume is $494 \mathrm{ml}$, standard liver volume is $1034 \mathrm{ml}$, left half liver / standard liver volume: $47.8 \%$, ECoG score is 0 ).

Because radical operation (plus lymph node dissection) is the only cure method for intrahepatic cholangiocarcinoma, it can be surgically removed after multidisciplinary evaluation. Group 16 lymph nodes were removed by exploratory laparotomy, and rapid frozen pathological found it was negative. It was evaluated that it could be surgically removed, so two months after the discovery of the tumor, right hemihepatectomy and cholecystectomy and porta hepatis, retroperitoneal lymph node dissection and abdominal tumor resection were performed. Pathological examination further confirmed

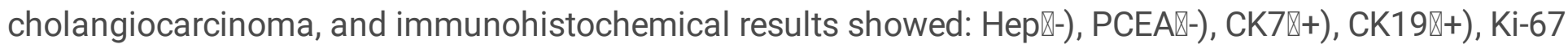
\$50\%), CEA囚-), CD10囚-邓(Fig. 4). Fifteen lymph nodes were removed from hepatoduodenal ligament and posterior edge of pancreas during the operation, 2 were found to be metastatic by pathological examinatio. The patient recovered well and was discharged from the hospital. Immunotherapy and GP chemotherapy were carried out one month after discharge.

After one year follow-up, no recurrence was found by CT imaging (Fig. 5) and CA199 decreased from $1844 \mathrm{u} / \mathrm{ml}$ to $4.76 \mathrm{u} / \mathrm{ml}$. As a result, oncology assessment reached PR status.

\section{Discussion}

Intrahepatic cholangiocarcinoma has high malignancy, poor prognosis and few long-term survivors. The prognosis of patients without operation is very poor, and the 3-year survival rate was only $40 \%-50 \%$ after the operation (5). Compared with other liver malignancies, ICC means shorter survival time, lower resection rate and cure rate (6). In addition, the gross classification of ICC is related to the prognosis of tumor. The prognosis of peritubular infiltration type is the worst, followed by mass type, and intratubular growth type is the best. At present, tumor markers lack sensitivity and specificity in the diagnosis of ICC. CA19-9 has a certain significance in the diagnosis of tumor, evaluation of tumor resection and prognosis, while CEA, AFP and CA-125 have little significance in the diagnosis of ICC(7). The definite diagnosis depends on the combination of imaging and pathological examination (8).

Intrahepatic cholangiocarcinoma is more likely to recur than hepatocellular carcinoma(9).The survival of patients with ICC is affected by the number of tumors, the extent of liver resection, the degree of tumor differentiation and the type of tumor cells. According to NCCN guidelines, preoperative biopsy is not necessary, especially in surgical resectable cases. Imaging suspicious nodules should be treated as 
malignant, and diagnostic laparoscopic exploration is recommended to exclude unresectable disseminated lesions (10).

Exploration and evaluation should include: multiple intrahepatic cancer, lymph node metastasis and distant metastasis. Lymph node metastasis and distant metastasis outside the porta hepatis should be considered as surgical contraindications. It is recommended that hilar lymph node dissection should be performed routinely to provide staging and prognosis information. According to NCCN guidelines, radical surgery (plus lymph node dissection) is the only cure treatment (11). Surgical indications and neoadjuvant therapy are still the focus of clinical justice (12). Literature reports showed that two patients with stage $\triangle B$ ICC received $13 \mathrm{~m}$ and $16 \mathrm{~m}$ CR after chemotherapy with PD-1 monoclonal antibody(13). This case of intrahepatic cholangiocarcinoma with hilar lymph node metastasis, abdominal aortic lymph node metastasis is suspected, belongs to AJCC stage $\otimes \mathrm{b}$. After multidisciplinary discussion, it is suggested that patients should implement neoadjuvant therapy and surgical resection and lymph node dissection and postoperative adjuvant therapy, in addition, neoadjuvant therapy includes chemotherapy and immunotherapy.

After immunotherapy with PD-1 and chemotherapy with GP regimen, re evaluation showed that the tumor was shrunk and necrotic, and the oncology evaluation reached PR. According to the NCCN guidelines, the patients continued to receive immunotherapy and chemotherapy after tumor resection. So far, no recurrence has been found. Lymph node dissection is another focus of clinical debate. The existing research data show that extended dissection does not improve the prognosis, however, both NCCN and ESMO guidelines recommend routine hilar lymph node dissection. NCCN guidelines consider that positive extrahepatic lymph node metastasis is a surgical contraindication, and neoadjuvant therapy is recommended, followed by surgery at a lower stage. So the independent risk factors for the prognosis of the patients included: the distance between the cutting edge and the tumor was less than $1 \mathrm{~cm}$; the diameter of the tumor was more than $5 \mathrm{~cm}$; the number of tumors was multiple; microvascular invasion and positive pathological lymph node metastasis $(12,14)$.

A literature confirmed that lymph node dissection cannot improve the prognosis of patients with intrahepatic cholangiocarcinoma(15). Postoperative pathology confirmed that positive lymph node metastasis is an independent risk factor affecting the prognosis of patients with intrahepatic cholangiocarcinoma, which confirmed the clinical value of lymph node dissection, and also showed that the current lymph node dissection are still many problems(15).Lymph node dissection is still routine (lymph node metastasis rate is $25-50 \%$ ), CSCO biliary system tumor diagnosis and treatment expert consensus 2019 recommend active surgical resection and lymph node dissection, so there is no consensus on the scope of regional lymph node dissection (14). We should carefully choose extended hepatectomy, the improvement of chemotherapy, the emergence of immunotherapy and radiotherapy With the development of surgery, adjuvant therapy has become an important means to improve the prognosis of intrahepatic cholangiocarcinoma $(16,17)$. 
Another point of contention about ICC is there is no RCT study on preoperative neoadjuvant therapy for ICC. A multicenter study contain 62 cases of ICC with neoadjuvant chemotherapy had a median OS of $47 \mathrm{~m}$ after resection, 74 cases of unresectable ICC had a median OS of $24 \mathrm{~m}$ after 6 cycles of chemotherapy(18). At present, preoperative neoadjuvant therapy was regarded as the "TEST", and there is no report about immunotherapy as neoadjuvant therapy or transformation therapy.

Immunotherapy as a research hotspot at present. PD1 inhibitors are effective for solid tumors, but currently only melanoma and lung cancer are the clinical indications for immunotherapy. At present, the objective remission rate of ICC in the treatment of advanced HCC patients is about $20 \%$, showing a certain efficacy, and more phase III clinical trials are needed to verify. The development of more sensitive and efficient predictive methods will help ICC therapy more accurately benefit potential patients with advanced HCC and benefit patients who are suitable for ICC therapy. This will hopefully open up a new prospect of immunotherapy for liver tumors (19). Immunotherapy may provide an attemptable treatment option for hepatic ICC, but it needs to be confirmed by a larger sample of cases (20); and as part of preoperative neoadjuvant therapy for intrahepatic cholangiocarcinoma is still a hot topic in clinical trials (21). Overall, the value of neoadjuvant therapy, the time of operation after neoadjuvant therapy, the necessity of lymph node dissection, the means of adjuvant therapy, and the treatment plan after recurrence are still the hot topics of current research.

\section{Conclusion}

Neoadjuvant therapy combined with surgical resection are useful for advanced stage intrahepatic cholangiocarcinoma. This is the first successful treatment case of stage Illb intrahepatic cholangiocarcinoma after neoadjuvant therapy with PD-1 blockade.

\section{Declarations}

\section{Author details}

${ }^{1}$ Department of Hepatic Surgery and Liver Transplantation, The Third Affiliated Hospital of Sun Yat-Sen University, Guangzhou 510000, Guangdong Province, China.

2 Department of Hepatobiliary Surgery of The First Affiliated Hospital of Guangzhou Medical University, Guangzhou 510000, Guangdong Province, China.

*Corresponding author.

Authors' contributions

GW drafted the manuscript. SZ collected the materials. HBL, JZ, CQ and HL were involved in the preoperative, intraoperative and postoperative management of the patient. TD provided the pathological figures. All authors read and approved the final manuscript. 
Competing interests

The authors declare that they have no competing interests.

Funding

This work was supported in part by the National 13th Five-Year Science and Technology Plan Major Projects of China (No.2017ZX10203205-006-001; 2017ZX10203205-001-003) and the Science and Technology Planning Project of Guangzhou (No. 158100076).

Ethics approval and consent to participate

Written informed consent was obtained from the patient for publication of this manuscript. Ethics approval was permitted by the Ethics committee for this research. The copy of the written consent and ethics approval are available for review by the Editor in Chief of this journal.

Consent for publication

Consent form is available upon request.

Availability of data and materials

All data generated or analysed during this study are included in this published article.

Acknowledgement

Not applicable.

\section{References}

1. Nuzzo G, Giuliante F, Ardito F, Giovannini I. Intrahepatic cholangiocarcinoma. Annals of surgery. 2009;249(3):541-2. doi:10.1097/SLA.0b013e31819aa76e. PubMed PMID: 19247046. author reply 2. Epub 2009/02/28.

2. Zhang H, Yang T, Wu M, Shen F. Intrahepatic cholangiocarcinoma: Epidemiology, risk factors, diagnosis and surgical management. Cancer letters. 2016;379(2):198-205. doi: 10.1016/j.canlet.2015.09.008. PubMed PMID: 26409434.

3. Rizvi S, Gores GJ. Pathogenesis, diagnosis, and management of cholangiocarcinoma. Gastroenterology. 2013;145(6):1215-29. doi:10.1053/j.gastro.2013.10.013. PubMed PMID: 24140396; PubMed Central PMCID: PMC3862291. Epub 2013/10/22.

4. Razumilava N, Gores GJ. Cholangiocarcinoma. Lancet (London. England). 2014;383(9935):216879. doi:10.1016/s0140-6736(13)61903-0. PubMed PMID: 24581682; PubMed Central PMCID:

PMC4069226. Epub 2014/03/04. 
5. Mavros MN, Economopoulos KP, Alexiou VG, Pawlik TM. Treatment and Prognosis for Patients With Intrahepatic Cholangiocarcinoma: Systematic Review and Meta-analysis. JAMA surgery. 2014;149(6):565-74. doi:10.1001/jamasurg.2013.5137. PubMed PMID: 24718873. Epub 2014/04/11.

6. Cillo U, Fondevila C, Donadon M, Gringeri E, Mocchegiani F, Schlitt HJ, et al. Surgery for cholangiocarcinoma. Liver international: official journal of the International Association for the Study of the Liver. 2019;39 Suppl 1(Suppl Suppl 1):143 - 55. Epub 2019/03/08. doi: 10.1111/liv.14089. PubMed PMID: 30843343; PubMed Central PMCID: PMC6563077.

7. Rahnemai-Azar AA, Weisbrod A, Dillhoff M, Schmidt C, Pawlik TM. Intrahepatic cholangiocarcinoma: Molecular markers for diagnosis and prognosis. Surg Oncol. 2017;26(2):125-37. doi:10.1016/j.suronc.2016.12.009. PubMed PMID: 28577718. Epub 2017/06/05.

8. Wang Y, Li J, Xia Y, Gong R, Wang K, Yan Z, et al. Prognostic nomogram for intrahepatic cholangiocarcinoma after partial hepatectomy. Journal of clinical oncology: official journal of the American Society of Clinical Oncology. 2013;31(9):1188-95. doi:10.1200/jco.2012.41.5984. PubMed PMID: 23358969. Epub 2013/01/30.

9. Chaisaingmongkol J, Budhu A, Dang H, Rabibhadana S, Pupacdi B, Kwon SM, et al. Common Molecular Subtypes Among Asian Hepatocellular Carcinoma and Cholangiocarcinoma. Cancer cell. 2017;32(1):57-70. doi:10.1016/j.ccell.2017.05.009. PubMed PMID: 28648284; PubMed Central PMCID: PMC5524207. e3. Epub 2017/06/27.

10. Cheng Z, Lei Z, Shen F. Coming of a precision era of the staging systems for intrahepatic cholangiocarcinoma? Cancer letters. 2019;460:10-7. doi:10.1016/j.canlet.2019.114426. PubMed PMID: 31212000. Epub 2019/06/19.

11. Idrees JJ, Merath K, Gani F, Bagante F, Mehta R, Beal E, et al. Trends in centralization of surgical care and compliance with National Cancer Center Network guidelines for resected cholangiocarcinoma. HPB: the official journal of the International Hepato Pancreato Biliary Association. 2019;21(8):981-9. doi: 10.1016/j.hpb.2018.11.013. PubMed PMID: 30591307.

12. Li T, Qin LX, Zhou J, Sun HC, Qiu SJ, Ye QH, et al. Staging, prognostic factors and adjuvant therapy of intrahepatic cholangiocarcinoma after curative resection. Liver international: official journal of the International Association for the Study of the Liver. 2014;34(6):953-60. doi:10.1111/liv.12364. PubMed PMID: 24134199. Epub 2013/10/19.

13. Sui M, Li Y, Wang H, Luo Y, Wan T, Wang $X$, et al. Two cases of intrahepatic cholangiocellular carcinoma with high insertion-deletion ratios that achieved a complete response following chemotherapy combined with PD-1 blockade. J immunother Cancer. 2019;7(1):125. doi:10.1186/s40425-019-0596-y. PubMed PMID: 31064408; PubMed Central PMCID: PMC6505215. Epub 2019/05/09.

14. Zhou R, Lu D, Li W, Tan W, Zhu S, Chen X, et al. Is lymph node dissection necessary for resectable intrahepatic cholangiocarcinoma? A systematic review and meta-analysis. HPB: the official journal 
of the International Hepato Pancreato Biliary Association. 2019;21(7):784-92.

doi:10.1016/j.hpb.2018.12.011. PubMed PMID: 30878490. Epub 2019/03/18.

15. Ji GW, Zhu FP, Zhang YD, Liu XS, Wu FY, Wang K, et al. A radiomics approach to predict lymph node metastasis and clinical outcome of intrahepatic cholangiocarcinoma. European radiology. 2019;29(7):3725-35. doi:10.1007/s00330-019-06142-7. PubMed PMID: 30915561. Epub $2019 / 03 / 28$.

16. Kelley RK, Bridgewater J, Gores GJ, Zhu AX. Systemic therapies for intrahepatic cholangiocarcinoma. Journal of hepatology. 2020;72(2):353-63. doi:10.1016/j.jhep.2019.10.009. PubMed PMID: 31954497. Epub 2020/01/20.

17. Valle JW, Lamarca A, Goyal L, Barriuso J, Zhu AX. New Horizons for Precision Medicine in Biliary Tract Cancers. Cancer discovery. 2017;7(9):943-62. doi:10.1158/2159-8290.cd-17-0245. PubMed PMID: 28818953; PubMed Central PMCID: PMC5586506. Epub 2017/08/19.

18. Rizvi S, Khan SA, Hallemeier CL, Kelley RK, Gores GJ. Cholangiocarcinoma - evolving concepts and therapeutic strategies. Nature reviews Clinical oncology. 2018;15(2):95-111. doi:10.1038/nrclinonc.2017.157. PubMed PMID: 28994423; PubMed Central PMCID: PMC5819599. Epub 2017/10/11.

19. Jing CY, Fu YP, Yi Y, Zhang MX, Zheng SS, Huang JL, et al. HHLA2 in intrahepatic cholangiocarcinoma: an immune checkpoint with prognostic significance and wider expression compared with PD-L1. J immunother Cancer. 2019;7(1):77. doi:10.1186/s40425-019-0554-8. PubMed PMID: 30885276; PubMed Central PMCID: PMC6421676. Epub 2019/03/20.

20. Lowery MA, Burris HA 3rd, Janku F, Shroff RT, Cleary JM, Azad NS, et al. Safety and activity of ivosidenib in patients with IDH1-mutant advanced cholangiocarcinoma: a phase 1 study. The lancet Gastroenterology \& hepatology. 2019;4(9):711 - 20. Epub 2019/07/14. doi: 10.1016/s24681253(19)30189-x. PubMed PMID: 31300360.

21. Lu JC, Zeng HY, Sun QM, Meng QN, Huang XY, Zhang PF, et al. Distinct PD-L1/PD1 Profiles and Clinical Implications in Intrahepatic Cholangiocarcinoma Patients with Different Risk Factors. Theranostics. 2019;9(16):4678-87. doi:10.7150/thno.36276. PubMed PMID: 31367249; PubMed Central PMCID: PMC6643449. Epub 2019/08/02.

\section{Figures}



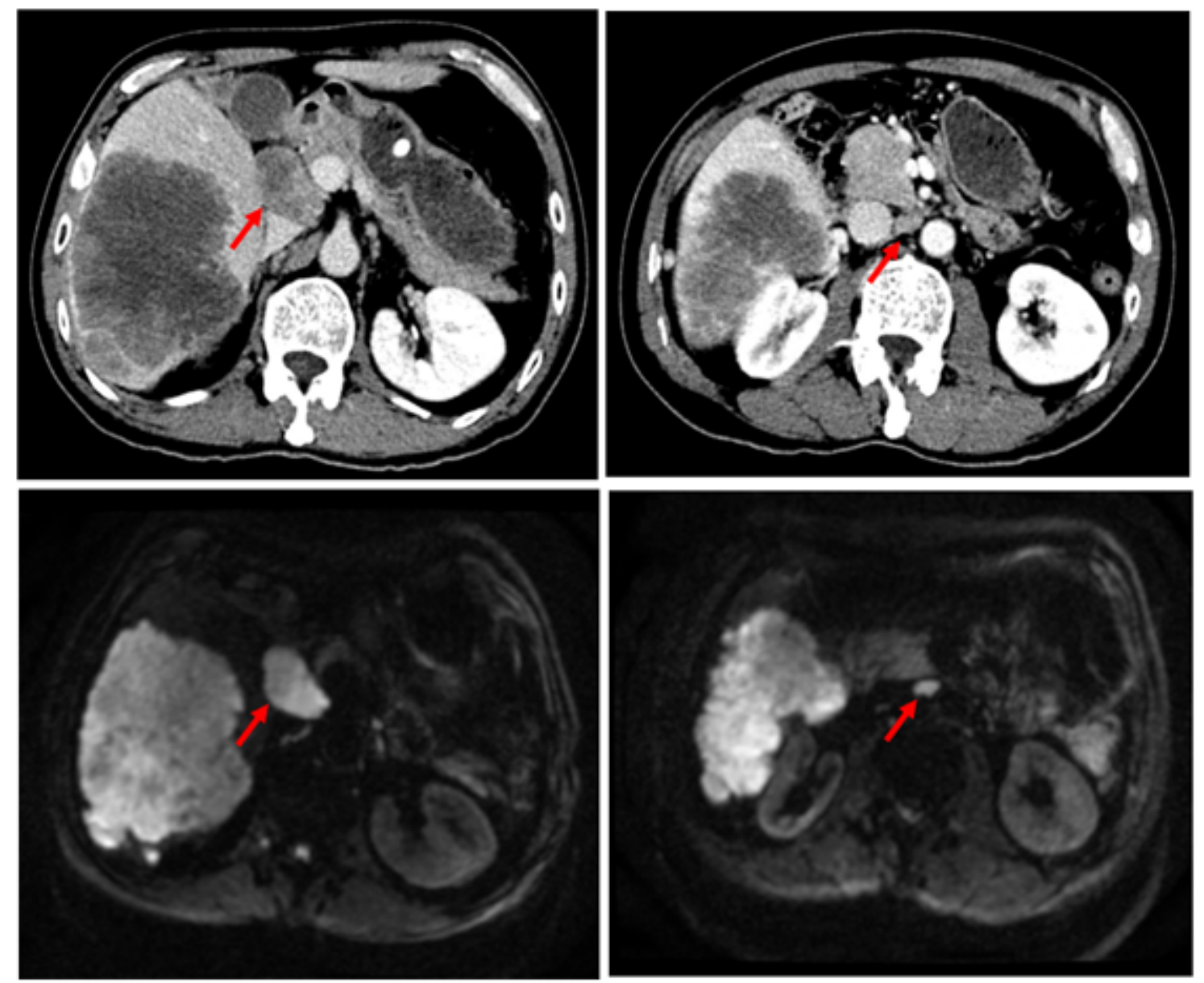

Figure 1

Computed tomography and Magnetic resonance imaging showed that the tumors were located in the right lobe, the longest diameter is about $20 \mathrm{~cm}$; hilar lymph node metastasis was found.

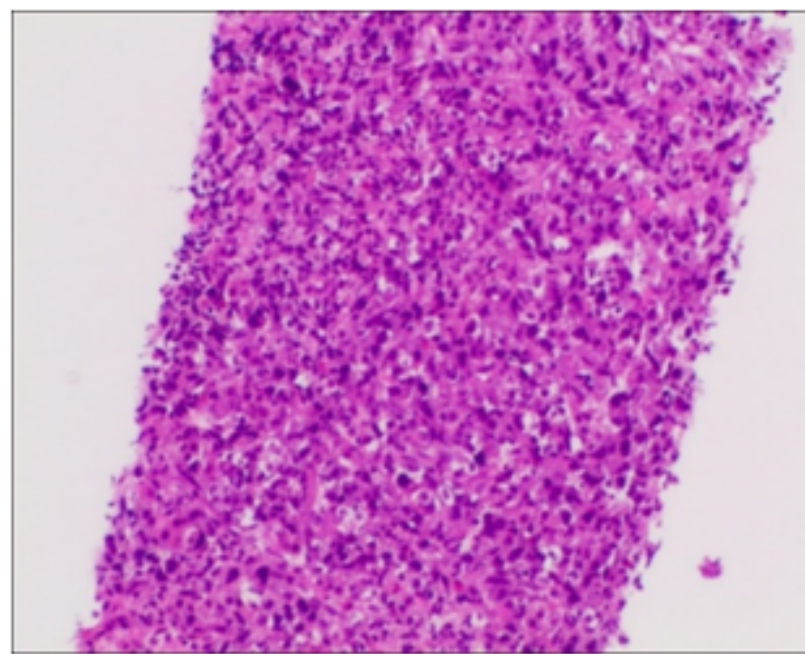

specimen from the biopsy

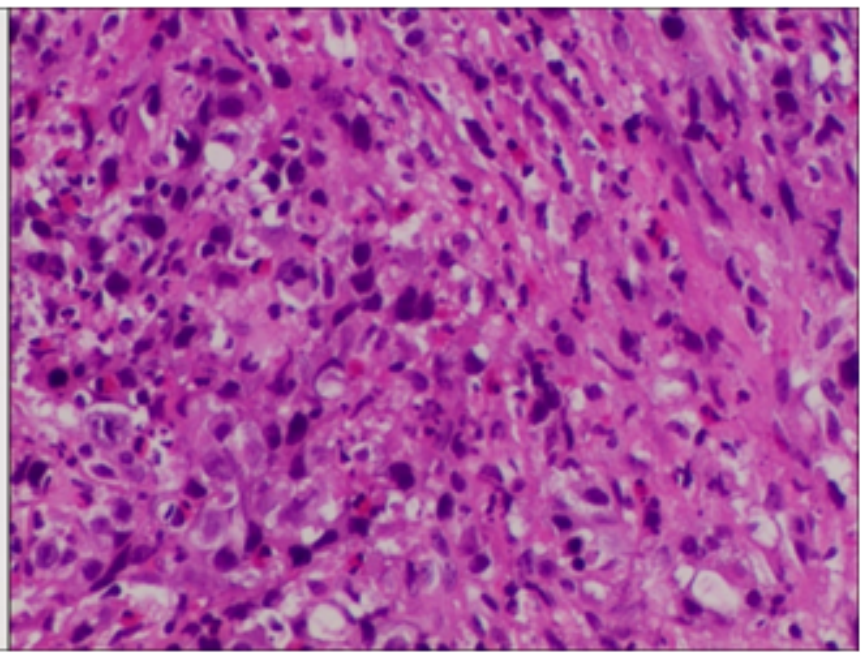

specimen $200 \mathrm{x}$

Figure 2 
The tumors showed fish-like changes on the whole, and atypical cells and lymphocyte infiltration could be seen under the microscope.
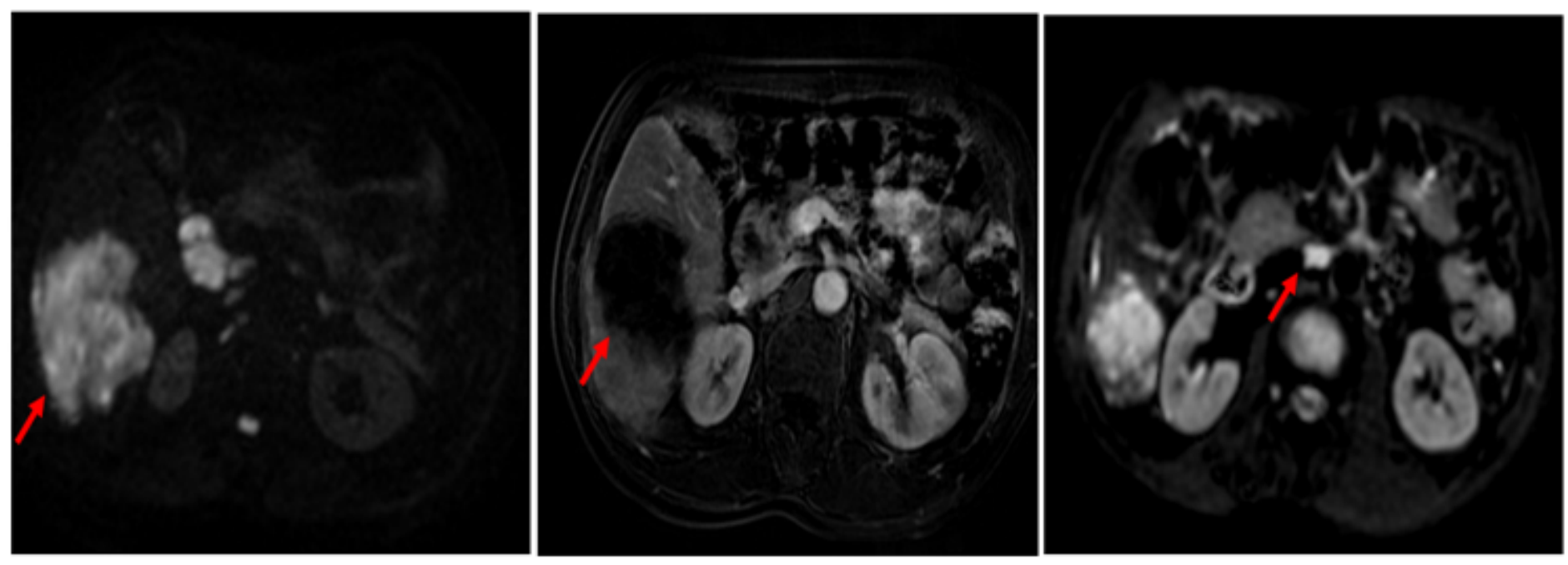

\section{Figure 3}

After neoadjuvant therapy, Magnetic resonance imaging showed the tumor size of the patients was reduced obviously and the hilar lymph node metastasis disappeared.

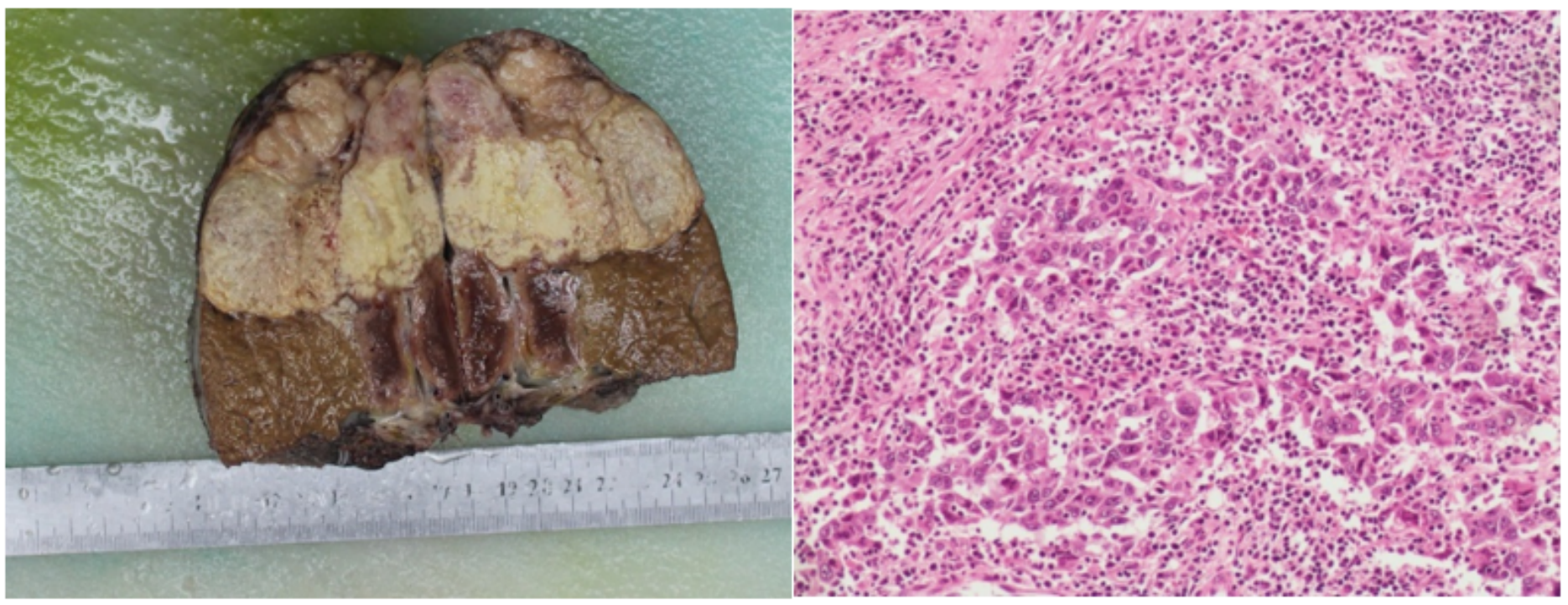

\section{Specimen from the Operation}

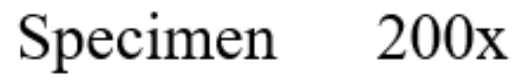

\section{Figure 4}

Samples after the operation and microscopically the same as the biopsy. 


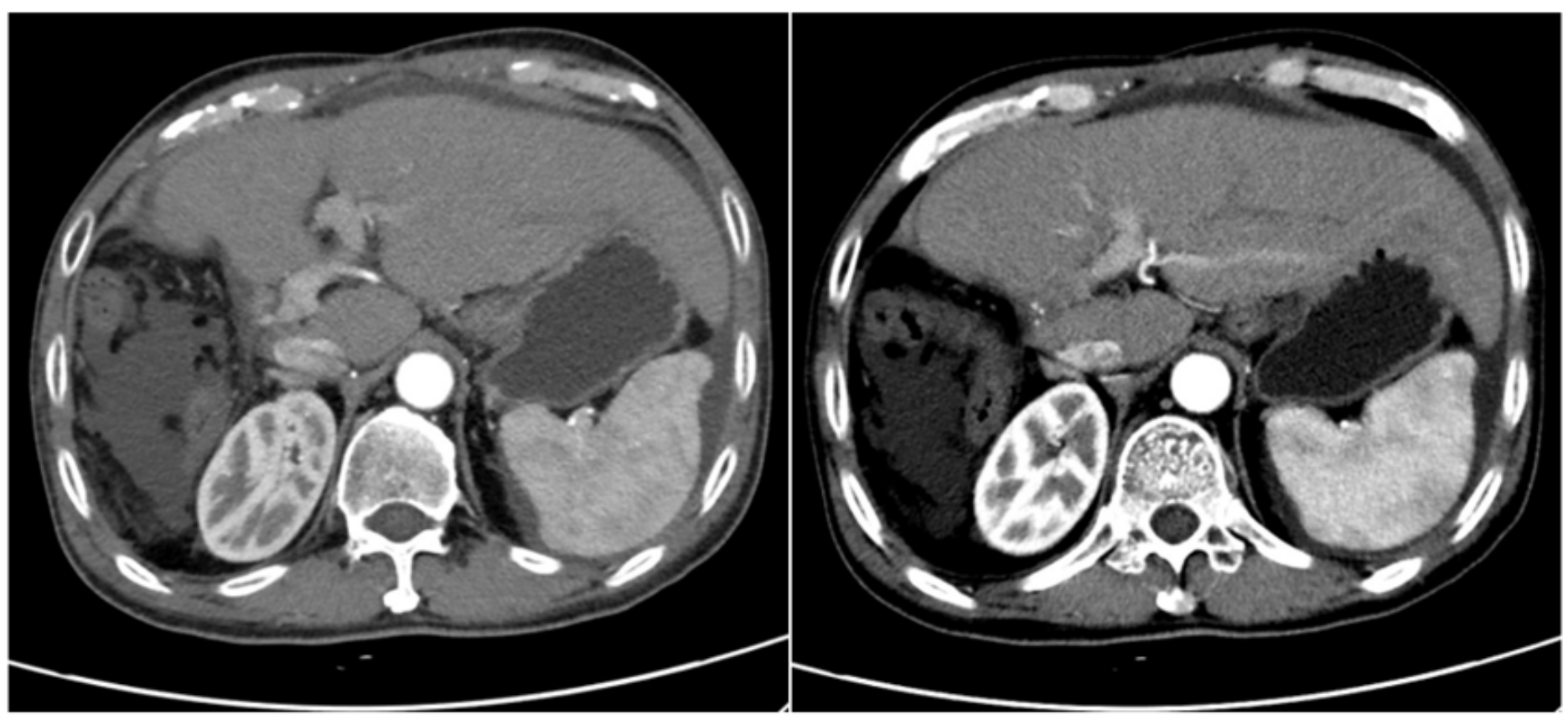

Figure 5

Eight months after the operation, no recurrence was found by CT scan. 